\title{
Deporte: modificaciones fisiológicas y evaluación para la prevención de la muerte súbita. (Parte I)
}

\author{
Dres. Alejandro Cuesta', Geraldine Rodríguez Estula², Sergio Giovanetti
}

\section{Resumen}

La práctica de deporte en forma regular ha demostrado ser beneficiosa para la salud y prolongar la vida en personas sanas, con patología cardiovascular (CV) y a cualquier edad. También es verdad que aumenta el riesgo de morir de forma súbita. Recientemente, ha surgido evidencia de que el exceso puede ser nocivo, y se continúa investigando. En los deportistas hay adaptaciones del aparato CV que pueden confundirse con anormalidades. También existen patologías CV que progresan más rápido con el ejercicio y anormalidades asintomáticas en las que el ejercicio puede desencadenar eventos adversos. La valoración predeportiva ha demostrado prevenir la muerte súbita (MS). Para no efectuar diagnósticos erróneos que afecten la vida de los deportistas, es necesario conocer las adaptaciones clínicas, electrocardiográficas y anatómicas que se consideran normales y usar pautas internacionales.

Se presenta una revisión que será publicada en dos partes. En la primera, tratamos el beneficio del deporte y las incertidumbres existentes en relación con el exceso de su práctica, así como al riesgo de MS, su fisiopatología y las ventajas de la valoración predeportiva. En la segunda entrega se abordarán los cambios clínicos, eléctricos y anatómicos adaptativos a la práctica del deporte y cómo identificarlos para una correcta habilitación.

Palabras clave: DEPORTE

DEPORTISTA

VALORACIÓN PREDEPORTIVA

\section{Sport: physiological modifications and evaluation for the prevention of sudden death. (Part I)}

\section{Summary}

The practice of regular sport has proven to be beneficial for health and prolong life, in healthy people, with cardiovascular pathology and at any age. It is also true that the risk of dying suddenly increases. Recently there has been evidence that the excess could be harmful and so research continues. In athletes there are adaptations of cardiovascular system that can be confused with abnormalities. There are also cardiovascular pathologies that progress faster with exercise and people with asymptomatic disorders in which exercise can trigger adverse events. Pre-sport assessment has been shown to prevent sudden death. In order to avoid erroneous diagnoses that affect athlete's life, it is necessary to know the clinical, electrocardiographic and anatomical adaptations that are considered normal and to use international guidelines.

A review is presented, which will be published in two parts. In the first one, the benefit of sport and uncertainties in relation to its excess are discussed, as well as the risk of sudden death, its pathophysiology and the advantage of pre-sport assessment. In the second part, the adaptive clinical, electrical and anatomical changes will be addressed and differentiated, for a correct qualification.

Key words: $\quad$ SPORT

ATHLETE

PRE-SPORT ASSESSMENT

1. Centro Cardiovascular Universitario, Hospital de Clínicas. Montevideo, Uruguay.

2. PREMUDE. Gol al Futuro, Secretaría Nacional del Deporte. Montevideo, Uruguay.

3. SUAT Emergencia Médica SC. Montevideo, Uruguay.

Correspondencia: Dr. Alejandro Cuesta. Correo electrónico: arritmia@yahoo.com.

Recibido Set 10, 2019; aceptado May 25, 2020. 


\section{Esporte: modificações fisiológicas e avaliação para prevenção de morte súbita. (Parte I)}

\section{Resumo}

A prática do esporte regular provou ser benéfica para a saúde e prolongar a vida, em pessoas saudáveis, com patologia cardiovascular e em qualquer idade. Também é verdade que aumenta o risco de morte súbita. Recentemente, houve evidências de que o excesso poderia ser prejudicial e está sendo investigado. Nos atletas, há adaptações do sistema cardiovascular que podem ser confundidas com anormalidades. Existem também patologias que progridem mais rapidamente com o exercício e pessoas com distúrbios assintomáticos nas quais o exercício pode desencadear eventos adversos. A avaliação pré-esportiva demonstrou prevenir a morte súbita. Para não fazer diagnósticos errôneos que afetam a vida dos atletas, é necessário conhecer as adaptações clínicas, eletrocardiográficas e anatômicas consideradas normais e utilizar diretrizes internacionais.

Se realiza uma revisão dividido em duas partes. Na primeira, é discutido o benefício do esporte e as incertezas em relação ao seu excesso, o risco de morte súbita, sua fisiopatologia e a vantagem da avaliação pré-esportiva. No segundo, será abordado as mudanças clínicas, elétricas e anatômicas adaptativas ao esporte e que devem ser distinguidas para uma correta habilitação.

Palavras chave: $\quad$ ESPORTE

ESPORTISTA

AVALIAÇÃO PRÉ-ESPORTE

\section{El deporte es salud}

La práctica deportiva es beneficiosa para la salud cardiovascular (CV). Las personas que realizan ejercicio viven más que las sedentarias ${ }^{(1)}$. Los deportistas tienen menor prevalencia de factores de riesgo CV, como hipertensión arterial (HTA) crónica, diabetes, dislipemia y obesidad ${ }^{(2)}$. También influye positivamente sobre otros sistemas y en la calidad de vida en general $^{(3)}$. Las ventajas no solo se dan en las personas jóvenes y sanas, también están demostradas en adultos mayores y con enfermedad vascular establecida, disminuyendo la incidencia de infarto de miocardio y muerte ${ }^{(4)}$. Atendiendo a lo anterior, es de buena práctica médica recomendar la realización de ejercicio físico, salvo circunstancias excepcionales.

En principio, cualquier nivel de ejercicio es beneficioso ${ }^{(5)}$. Así se afirma en la última guía del Colegio Americano de Cardiología (ACC, por su sigla en inglés) para el manejo del estilo de vida y la disminución del riesgo $\mathrm{CV}^{(6)}$. Un metaanálisis concluyó que es mejor hacer al menos un poco de ejercicio que nada y que hay una relación directa entre la intensidad del ejercicio y el beneficio que se obtiene ${ }^{(7)}$. Incluso se han demostrado ventajas en la sobrevida global en deportistas de muy alta exigencia y rendimiento ${ }^{(8)}$. Este último concepto, sostenido por mucho tiempo, está siendo cuestionado en los últimos años.

\section{Cuestionamientos al exceso de ejercicio}

Solemos pensar de forma binaria, en términos de corazón sano y corazón enfermo, pero la realidad es mucho más compleja y hay al menos otras tres situaciones a considerar.

a) Existen sujetos sanos que tienen adaptaciones CV al deporte difíciles de diferenciar de la enfermedad. Es el denominado corazón del deportista. A veces resulta necesario profundizar la observación, para diferenciar la enfermedad de la adaptación. Los errores en este aspecto afectan la calidad de vida de la persona y ocasionan un gasto innecesario de recursos sanitarios.

b) Existen personas sin alteraciones estructurales cardíacas (fenotipo), pero con predisposición genética para desarrollar determinadas enfermedades y el deporte podría acelerar esta expresión. La miocardiopatía arritmogénica del ventrículo derecho (MAVD) es el mejor ejemplo de esto. Inicialmente se demostró en modelos animales y luego se planteó también en huma$\operatorname{nos}^{(9,10)}$. Algo similar se ha observado con la aterosclerosis. Si bien los deportistas tienen globalmente menos enfermedad coronaria porque tienen menos factores de riesgo, si comparamos deportistas y no deportistas con el mismo puntaje de riesgo, los primeros tienen paradójicamente más aterosclerosis ${ }^{(11)}$.

c) Por último, determinados ejercicios o su intensidad podrían generar sustratos arrítmicos. En ratas sanas sometidas a ejercicio intenso se observó remodelación de cavidades, pero además fibrosis miocárdica. Esto se acompañó de una mayor inducibilidad de arritmias ventriculares $^{(12)}$ y se demostró que favorecía la fibrilación auricular (FA)(13). En un estudio epidemiológico de casos y controles se encontró que, entre otros factores, el entrenamiento vigoroso de resisten- 
cia se asociaba a la ocurrencia de FA, mientras que grados menores protegían contra esta. Se describió una curva en U de la que tantos ejemplos encontramos en medicina ${ }^{(14)}$. En otro estudio epidemiológico se describió una relación similar para ejercicio, enfermedad coronaria, cerebrovascular y tromboembolismo venoso ${ }^{(15)}$. Ningún trabajo ha sido concluyente, pero encienden una luz de advertencia. Ya no es tan claro que cuanto más ejercicio se realice, siempre será mejor para la salud. Los sujetos que desarrollan ejercicio intenso por períodos prolongados, deben ser valorados muy cuidadosamente.

Deporte y riesgo de muerte súbita: definiciones, incidencia y causas

La práctica de deportes es saludable y debe ser recomendada, pero se ha demostrado que en determinadas circunstancias puede aumentar el riesgo de muerte súbita (MS). Se han propuesto diferentes definiciones de MS, existiendo otros términos que se emplean de forma similar y se prestan a confusión. Esto debe tenerse en cuenta al comparar distintos trabajos.

Se entiende como MS a la muerte no traumática, inesperada, que ocurre en menos de una hora de iniciado cualquier síntoma en un sujeto aparentemente sano. Cuando no hay testigos, se puede considerar MS si la persona fue vista en buen estado de salud durante las 24 horas previas al evento.

Para limitar la MS a la enfermedad cardíaca, se denomina MSC. Se define de la misma manera, incluyendo a las personas en que se conocía la existencia de una enfermedad cardíaca previa, aunque el momento de la muerte sea inesperado ${ }^{(16)}$. Numerosos estudios epidemiológicos de MS en deportistas incluyen la ocurrencia del evento en cualquier momento. Otros trabajos, solo cuando el evento tiene lugar durante o hasta una hora después de realizar ejercicio. En el primer caso se habla de MS en el deportista en general y en el segundo, MS asociada al deporte.

Los trabajos que analizan las causas de MS cuando la autopsia y el análisis toxicológico no son concluyentes y el corazón es estructuralmente normal, usan la denominación de MS arrítmica. Con ello se quiere expresar la sospecha de una causa arrítmica genética primaria, aunque se puede inducir a considerar erróneamente que las demás muertes no fueron arrítmicas.

El término MS inexplicada se utiliza cuando la muerte no tiene causa aparente y no se ha realizado la autopsia. A los efectos clínicos y epidemiológicos aporta muy poco, y lamentablemente es frecuente.
Otro elemento a considerar en la comparación de trabajos, es el tipo de evento incluido. Hay algunos que incluyen solo a los deportistas fallecidos y otros que incluyen a los que sobreviven a un paro cardíaco. En los lugares con personal entrenado en soporte vital básico y acceso público rápido a desfibriladores, estas poblaciones son diferentes, dado que el número que sobrevive a un paro cardíaco es cada vez mayor.

En el año 2003, Corrado y colaboradores demostraron de forma concluyente que en adolescentes y jóvenes deportistas el riesgo de MS era entre 1,8 y 3,4 veces mayor que entre los no deportistas. También mostraron que el riesgo mayor se debía al aumento de los casos de MS en los que luego se identificaban causas $\mathrm{CV}^{(17)}$.

La incidencia de MS en deportistas varía en los distintos reportes. El mismo grupo italiano que en 2003 informó una prevalencia de 2,3 casos $/ 100.000$ atletas al año ${ }^{(17)}$, había comunicado anteriormente 1,6/100.000, considerando la misma población ${ }^{(18)}$. Maron y colaboradores encontraron en estudiantes de Estados Unidos una incidencia menor: 0,46/100.000 por año(19). Más recientemente, el mismo grupo, en el mayor estudio epidemiológico realizado en la materia, informó una incidencia de $0,61 / 100.000$ deportistas por año ${ }^{(20)}$. No se encontraron datos a nivel nacional, pero a los efectos prácticos se toma como referencia 1 caso/100.000 atletas por año.

Según cifras del Instituto Nacional de Estadística del Uruguay, en el año 2018 la tasa de mortalidad global anual fue 9,4/1.000; la mortalidad infantil 9,6/10.000 nacidos vivos, y las muertes por accidentes de tránsito 1,5/10.000 habitantes ${ }^{(21)}$. Tomando estas tasas como referencia, se observa que la incidencia de MS asociada al deporte es baja. Pero desde un punto de vista simbólico, las cifras resultan impactantes para la sociedad. Fallecen personas que son referencia para otros deportistas, paradigmas de la salud, y con enorme cobertura mediática. Esto compromete la prédica sobre las bondades del deporte y la eficacia de las medidas preventivas.

Las causas que explican la MS en deportistas son diversas y varían de acuerdo a la población que se estudie: dependen del país, composición étnica, grupo etario y características de los controles practicados a los deportistas para ser habilitados.

En la región del Véneto, Italia, las causas más comunes de MS en atletas fueron la MAVD (22\%), la aterosclerosis coronaria prematura $(18 \%)$ y el origen anómalo de arteria coronaria (12\%). La miocardiopatía hipertrófica ( $\mathrm{MCH}$ ) solo se encontró en $2 \%$ de los casos de MS de atletas y en 7\% de las MS de no atletas. Por otro lado, la MCH fue de las principales 
causas para la descalificación en la valoración pre$\operatorname{deportiva}^{(17)}$.

En Estados Unidos la importancia relativa de cada una de las causas es variable. El 85\% de las MS se explicó por causa CV y del resto, la mayoría fue por traumatismos cardíacos a tórax cerrado (commotio cordis). El $90 \%$ eran varones y esta predominancia masculina es aceptada por todos los autores. Las enfermedades CV identificadas en autopsias como causa de la muerte fueron, en primer lugar $\mathrm{MCH}(36 \%)$, y luego las malformaciones coronarias (13\%), seguidas de miocarditis, aneurisma y estenosis aórtica. Se reportó MAVD en menos del $3 \%$ de los $\operatorname{casos}^{(22,23)}$. La MCH fue una de las causas más frecuentes, aun en la población afroamericana, a pesar de que en esta, el diagnóstico clínico es menos frecuente $^{(24)}$.

En el año 2009 se publicó la serie más importante con un total de 1.866 atletas que fallecieron de forma súbita o sobrevivieron a un paro cardíaco ${ }^{(20)}$. La edad media fue de 19 años y $89 \%$ eran hombres. El 56\% de los casos se adjudicaron a enfermedades CV, $22 \%$ a un trauma contundente que causó daño estructural, $3 \%$ a commotio cordis y $2 \%$ a golpe de calor. De las 1.049 muertes CV, 690 fueron adjudicadas de forma confiable a 44 enfermedades CV primarias. Las más comunes fueron $\mathrm{MCH}$ en $36 \%$, anomalías congénitas de arteria coronaria en 17\%, miocarditis 6\% y MAVD 4\%. En $4 \%$ se realizó diagnóstico de enfermedad cardíaca arrítmica primaria, por existir registros electrocardiográficos previos o posteriores al evento, o por el estudio genético. Otras causas fueron enfermedad aterosclerótica coronaria, rotura de aorta y enfermedad de Wolf-Parkinson-White ${ }^{(20)}$.

Los datos epidemiológicos de España provienen de un solo estudio que analiza un número importante de MS asociadas al deporte, incluyendo todas las edades y circunstancias. La primera causa fue la coronariopatía adquirida con $41 \%$, pero allí se agrupan los sujetos de mayor edad, entre 28 y 65 años. La segunda causa fue la MCH (16\%), seguida de la MAVD (7\%). Hubo 16\% de causa indeterminada, y si se consideran solo los menores de 30 años, el $30 \%$ quedó sin diagnóstico ${ }^{(25,26)}$. Se plantea que en ese grupo estén probablemente los sujetos con enfermedades genéticas arrítmicas que no tienen expresión anatómica, las denominadas canalopatías.

\section{Fisiopatología de la muerte súbita}

El deporte favorecería la ocurrencia de MS mediante la aceleración de procesos patológicos ya existentes u oficiando de disparador de la complicación final. El primer punto ya fue desarrollado al analizar las evidencias de la progresión de la MAVD y de la calcificación coronaria; nos centraremos ahora en el deporte como "gatillo".

El deporte determina estrés físico y emocional importantes, de mayor magnitud en el deporte competitivo o cuando se exige un logro, una meta, o una marca, para obtener la recompensa. También incluye a los estudiantes de secundaria, a los que se evalúa curricularmente en base a marcas, y a deportistas aficionados.

Durante el ejercicio aeróbico y anaeróbico, de distinta manera, se instalan condiciones hemodinámicas mucho más exigentes que las basales. Esto involucra las variables: frecuencia cardíaca, volumen de eyección (gasto cardíaco), resistencias pulmonares y sistémicas.

El sistema nervioso autónomo experimenta importantes modificaciones al momento del ejercicio y también a largo plazo. El predominio adrenérgico durante las pruebas contrasta con el aumento del tono vagal en reposo. La transición de una a otra situación en el inicio brusco del ejercicio y en el posesfuerzo inmediato exige mecanismos muy eficientes de compensación, entre la frecuencia, el gasto y el sistema vasomotor.

Las altas exigencias que sufre el miocardio y la presencia de mediadores adrenérgicos son prácticamente un modelo proarrítmico experimental. Es similar a lo que se utiliza en el laboratorio de electrofisiología cuando se realizan estudios de inducción. Puede, además, determinar la ocurrencia de isquemia miocárdica con liberación de enzimas específicas aun en ausencia de lesiones coronarias, que ha demostrado ser indicadora de eventos adversos posteriores $^{(27,28)}$.

Hay deportes que implican la exposición a ambientes con condiciones de temperatura y humedad muy alejados de las condiciones fisiológicas. Se obliga al organismo a trabajar intensamente en condiciones alejadas de la homeostasis interna y aun en hipertermia. Ello requiere de un esfuerzo mayor de todos los sistemas que mantienen ese equilibrio.

La exigencia también se relaciona con la pérdida de agua y electrolitos. Hay mayores pérdidas durante la realización de ejercicios continuos por tiempo prolongado. Se han elaborado pautas de reposición para distintos deportes, pero se aplican solo en actividades y competencias que cuentan con medios para su instrumentación. Lamentablemente -la mayoría de las veces-, queda a criterio o posibilidad del atleta participante.

Otra variable a considerar es la presencia de drogas ilícitas o sustancias prohibidas en los atletas. La lista está bien definida por la Agencia Mundial Antidopaje, que la actualiza anualmente. Incluye esteroides androgénicos anabolizantes, hormonas, 
agonistas adrenérgicos y beta-bloqueantes, narcóticos, particularmente cocaína, cannabinoides, glucocorticoides y alcohol, entre otras. Casi todas las drogas ilícitas son potencialmente arritmogénicas incluso en ausencia de un sustrato orgánico o genético previo $^{(29)}$.

Prácticamente todas las causas cardíacas, el commotio cordis y las mediadas por drogas tienen una vía final común que es la ocurrencia de una arritmia ventricular sostenida que determina el paro hemodinámico y la MS. Las causas CV que no tienen esta vía final son el taponamiento cardíaco, las roturas vasculares, el tromboembolismo masivo y otras patologías traumáticas, que son mucho menos frecuentes. Este es un hecho muy importante a considerar a la hora de planificar y realizar la asistencia.

\section{Prevención de la muerte súbita}

En prevención primaria nos referimos al conjunto de medidas a tomar para evitar que ocurra el evento, y en prevención secundaria a las medidas que deben estar dispuestas previamente para que en caso de MS, el deportista pueda ser rescatado y no ocurra su muerte definitiva.

En cuanto a la prevención secundaria, podemos evitar que el deportista muera si es reanimado de forma rápida y efectiva. Para ello, es imprescindible contar con personal entrenado en maniobras de resucitación cardiopulmonar básica y tener acceso rápido a la desfibrilación eléctrica. Se debería contar con un desfibrilador externo automático en los lugares donde haya concentración de deportistas o público.

En Uruguay, la ley $\mathrm{N}^{\circ} 18.360$, promulgada el 26 de setiembre del año 2008 y reglamentada el 21 de julio de 2009, establece pautas para ambas medidas de prevención. Se ha trabajado mucho desde el Consejo Nacional de Resucitación, la Comisión Honoraria para la Salud Cardiovascular y la Sociedad Uruguaya de Cardiología. Igualmente, estamos lejos de que la ley se aplique universalmente, ya que no se ha instrumentado un sistema de fiscalización y ha quedado librado a la buena voluntad de las instituciones $^{(30,31)}$.

En prevención primaria debemos considerar varios aspectos. Uno es tener ámbitos deportivos seguros, ropa y sistemas de protección adecuados para el tipo de deporte que se practica. Esto requiere de pautas obligatorias y mecanismos de control que actualmente no existen.

Debe considerarse el estado físico y el nivel de entrenamiento requerido para la intensidad de ejercicio que se va a realizar. En este aspecto, tanto el deportólogo como los profesores de educación física tienen un rol fundamental. Existe evidencia sobre los efectos nocivos en el denominado "deportista de fin de semana", o de intentar desafíos que no se condicen con el nivel de ejercicio habitual. Un buen ejemplo de esto son las carreras de resistencia que se han puesto de moda, como eventos promocionales de marcas comerciales y en donde no se exige ninguna habilitación a los participantes.

Otro aspecto importante es la alimentación y los hábitos saludables. Ello incluye el aporte adecuado, en cantidad y calidad, preparatorio para la competencia y regenerativo. También el descanso posterior y el tiempo de recuperación entre actividades. Evitar el uso de drogas ilegales, alcohol, tabaco y algunos medicamentos que están indicados en condiciones especiales, pero que pueden afectar la salud del deportista.

\section{Valoración predeportiva}

Muchos deportistas que fallecen de forma súbita tienen enfermedades que hubieran podido detectarse en una valoración médica previa.

En 1996, la Asociación Americana del Corazón (AHA) publicó una declaración en la que por primera vez se realizaron recomendaciones institucionales sobre la valoración predeportiva ${ }^{(32)}$. Los autores manejaron conceptos de índole ético, legal y médico, respaldándose en la poca evidencia que existía. La declaración establecía que la evaluación CV era un objetivo alcanzable y debía ser obligatoria para todos los atletas, recomendando efectuar la historia clínica y el examen físico a todos los jóvenes en secundaria y universidad, antes de participar en deportes. Esa evaluación debía repetirse cada dos años, aunque poco después se publicó una modificación aumentando el intervalo a cuatro años en estudiantes de secundaria(33). Se aceptaba que la historia y el examen físico estándar carecen de capacidad para identificar de manera confiable muchas anormalidades CV potencialmente letales ${ }^{(32)}$. Consideraban que las pruebas no invasivas podían mejorar el poder diagnóstico, pero que no era prudente recomendar el uso rutinario de electrocardiograma (ECG), ecocardiograma o ergometría a grandes poblaciones, por el "bajo rendimiento" que tendrían estas técnicas. Existía la preocupación de que pudieran presentarse muchos falsos positivos, causar ansiedad innecesaria y exclusión injustificada de cobertura de seguros de salud y de la competencia atlética.

Se consideraba también que era prudente realizar selectivamente pruebas de esfuerzo en hombres mayores de 40 años y en mujeres mayores de 50 
años que desearan participar en entrenamiento físico regular y en deportes competitivos, si el médico sospechaba una enfermedad coronaria por la presencia de varios factores de riesgo o un único factor marcadamente anormal.

Resulta llamativo que el primer autor de la declaración y su grupo publicaron el mismo año un estudio epidemiológico retrospectivo sobre 134 atletas escolares y universitarios que sufrieron MS. De ellos, 115 habían tenido una evaluación médica previa solo con interrogatorio y examen físico, habiéndose sospechado enfermedad CV en cuatro (3\%), y solo en uno la anomalía CV responsable de la $\mathrm{MS}^{(34)}$. Se estaba implementando una guía que, según su propia experiencia, iba a ser capaz de evitar una muerte cada 134 deportistas.

En el año 2005 se publicó el consenso de la Sociedad Europea de Cardiología (ESC), manteniendo lo declarado por la AHA en cuanto a que la valoración preparticipativa de atletas jóvenes competitivos era justificable y conveniente por motivos éticos, legales y médicos. Pero, a diferencia de la pauta estadounidense, recomendaba un protocolo de evaluación que incluía la realización de rutina de un ECG de 12 derivaciones, única modalidad de evaluación que había demostrado efectividad, particularmente en la identificación de atletas con MCH. Casi toda la guía se basaba en la experiencia italiana ${ }^{(35)}$. Establecían que el ECG puede detectar alteraciones que hagan sospechar MCH, MAVD, síndrome QT largo y QT corto, síndrome de Brugada, preexcitación ventricular y enfermedad de Lenègre. La suma de todas estas entidades representaría el $60 \%$ de las causas de MS en deportistas. Incluyeron además, criterios para considerar un ECG anormal. Propusieron que el control debía comenzar al inicio de la actividad atlética competitiva, que en la mayoría de los deportes está entre los 12 y 14 años, y que debía repetirse de forma regular cada dos años ${ }^{(35)}$.

En 1998, Corrado y colaboradores informaron sobre su experiencia de 17 años en el Centro de Medicina Deportiva de Padua. Analizaron 34.000 atletas menores de 35 años que habían recibido una valoración predeportiva, incluyendo ECG. El 3\% había sido descalificado para competir: 59\% de ellos por una anormalidad CV, $13 \%$ ortopédica, $12 \%$ oftálmica, $4 \%$ neurológica, $4 \%$ respiratoria, $3 \%$ nefrológica y $5 \%$ otras. Las anormalidades CV más frecuentes fueron alteraciones del ritmo y la conducción, HTA y MCH. La mayoría de las alteraciones se encontraron en el $\mathrm{ECG}^{(19)}$. En el mismo período de 17 años se registraron 49 casos de MSC en atletas competitivos. Las causas más comunes fueron: MAVD en $22 \%$, aterosclerosis coronaria en $18 \%$ y origen anómalo de arteria coronaria en $12 \%$. Hubo una sola muerte relacionada con $\mathrm{MCH}$. Ninguno de los descalificados por MCH sufrió MSC en el seguimiento a $8 \pm 5$ años ${ }^{(19)}$.

En 2006, el mismo grupo presentó la información a 26 años de inicio del programa de evaluación predeportiva $^{(36)}$. La incidencia de MS en atletas había disminuido 89\%. Expresada en tasas descendió de 3,6/100.000 personas por año en 1979-1980 a 0,4/100.000 personas por año en 2003-2004. Esto ocurrió mientras la incidencia de MS en la población general no cambió significativamente. El descenso de la mortalidad comenzó claramente luego de que se implementó el control obligatorio. El costo estimado en Italia fue de 40 dólares por deportista y fue considerado aceptable para ese sistema de sa$\operatorname{lud}^{(20)}$.

La respuesta de la AHA no se hizo esperar y en 2007 actualizó sus recomendaciones planteando a texto expreso la discusión con la pauta europea(37). Se mantuvo el criterio de incluir solo el interrogatorio y el examen físico como pautas obligatorias, considerando que la estrategia europea era muy difícil de instrumentar en Estados Unidos, por diversas razones, y que a pesar de carecer de estudios de costo-beneficio, el costo sería muy alto. La evaluación individual con ECG podría significar el doble del costo estimado por los europeos. También se consideró problemático excluir al resto de la población con el único argumento de haber escogido no realizar ejercicio. Por otra parte, carecían de la infraestructura y los recursos humanos necesarios ${ }^{(37)}$, a pesar de que las evaluaciones son realizadas por médicos generales o profesionales no médicos. En ningún momento se planteó disminuir costos como eventual solución.

El principal aporte de esta guía consistió en sistematizar la clínica en 12 puntos "de alerta”, ocho del interrogatorio y cuatro del examen físico, que en caso de ser positivos debían determinar una evaluación CV más profunda, conocidos como los "12 pasos de la AHA"(37) (tabla 1).

En el año 2017, la Asociación Europea del Ritmo Cardíaco (EHRA), capítulo de la ESC, publicó su posición sobre el tema, con la adhesión de las sociedades equivalentes, asiática (AP-HRS), latinoamericana (LAHRS) y norteamericana (HRS) ${ }^{(38)}$. Se especifica que la evaluación predeportiva a la que se refieren involucra a los individuos de cualquier edad, que realizan ejercicio de entrenamiento regular, tanto en forma aficionada como profesional, independientemente del estatus competitivo. Hace una revisión exhaustiva de los argumentos a favor y en contra del ECG, incluyendo un metaanálisis recien$t^{(39)}$. Finalmente, en las recomendaciones afirma que la inclusión del ECG en los protocolos aumenta 
Tabla 1. Doce pasos de la AHA para la valoración clínica predeportiva ${ }^{(39)}$.

Historia personal

1. Dolor/malestar de esfuerzo en el pecho

2. Síncope/presíncope sin explicación

3. Disnea/fatiga de esfuerzo excesiva e inexplicada

4. Reconocimiento previo de un soplo cardíaco

5. Presión arterial sistémica elevada

Historia familiar

6. Muerte antes de los 50 años debido a una enfermedad cardíaca

7. Discapacidad por enfermedad cardíaca en familiar cercano menor de 50 años

8. Familiar con MCH o dilatada, síndrome de QT largo u otra canalopatía, síndrome de Marfan o arritmias importantes

Examen físico

9. Soplo cardíaco

10. Pulsos femorales para excluir la coartación aórtica

11. Estigmas físicos del síndrome de Marfan

12. Presión arterial de la arteria braquial (posición sentada)

AHA: American Heart Association; $\mathrm{MCH}$ : miocardiopatía hipertrófica.

de forma sustancial la capacidad diagnóstica. Para el análisis del ECG recomienda los denominados criterios de Seattle, y no los de la ESC, descritos anteriormente ${ }^{(35,40,41)}$. Agrega que el ecocardiograma de rutina no suma información para el diagnóstico y no resulta costo- efectivo ${ }^{(38)}$.

\section{Alejandro Cuesta, \\ https://orcid.org/0000-0003-1315-5581 \\ Geraldine Rodríguez Estula, \\ https://orcid.org/0000-0001-6326-524X \\ Sergio Giovanetti, \\ https://orcid.org/ 0000-0003-0574-3658}

Este artículo fue aceptado para su publicación por: Editora anterior Dra. María del Pilar Aguilar.

\section{Bibliografía}

1. Jonker JT, De Laet C, Franco OH, Peeters A, Mackenbach J, Nusselder WJ. Physical activity and life expectancy with and without diabetes: life table analysis of the Framingham Heart Study. Diabetes Care 2006;29(1):38-43. doi: 10.2337/diacare.29. 01.06.dc05-0985

2. Rodriguez BL, Curb JD, Burchfiel CM, Abbott RD, Petrovitch H, Masaki K, et al. Physical activity and 23-year incidence of coronary heart disease morbidity and mortality among middle-aged men. The Honolulu Heart Program. Circulation 1994;89 (6):2540-4. doi:10.1161/01.CIR.89.6.2540

3. Bize R, Johnson JA, Plotnikoff RC. Physical activity level and health-related quality of life in the general adult population: a systematic review. Prev Med.2007;45(6):401-15. doi: 10.1016/j.ypmed.2007. 07.017

4. Thompson PD, Buchner D, Pina IL, Balady GJ, Williams MA, Marcus BH, et al. Exercise and physical activity in the prevention and treatment of atherosclerotic cardiovascular disease: a statement from the Council on Clinical Cardiology (Subcommittee on Exercise, Rehabilitation, and Prevention) and the Council on Nutrition, Physical Activity, and Metabolism (Subcommittee on Physical Activity). Circulation 2003;107(24):3109-16. doi: 10.1161/01.CIR. 0000075572.40158 .77

5. Shiroma EJ, Lee IM. Physical activity and cardiovascular health: lessons learned from epidemiological studies across age, gender, and race/ethnicity. Circulation 2010;122(7):743-52. doi: 10.1161/CIRCULATIONAHA. 109.914721

6. Eckel RH, Jakicic JM, Ard JD, de Jesus JM, Houston Miller N, Hubbard VS, et al. 2013 AHA/ACC guideline on lifestyle management to reduce cardiovascular risk: a report of the American College of Cardiology/American Heart Association Task Force on Practice Guidelines. J Am Coll Cardiol. 2014;63 (25 Pt B):2960-84. doi: 10.1016/ j.jacc.2013.11.003

Fe de errata: J Am Coll Cardiol. 2014 Jul 1;63(25 Pt B):3027-8

7. Sattelmair J, Pertman J, Ding EL, Kohl HW 3rd, Haskell W, Lee IM. Dose response between physical activity and risk of coronary heart disease: a meta-analysis. Circulation 2011;124(7):789-95. doi:10.1161/CIRCULATIONAHA.110.010710

8. Marijon E, Tafflet M, Antero-Jacquemin J, El Helou N, Berthelot G, Celermajer DS, et al. Mortality of French participants in the Tour de France (1947-2012). Eur Heart J. 2013;34(40):3145-50. doi: 10.1093/eurheartj/eht347

9. Kirchhof $\mathbf{P}$, Fabritz L, Zwiener M, Witt $\mathbf{H}$, Schäfers M, Zellerhoff S, et al. Age- and training-dependent development of arrhythmogenic right 
ventricular cardiomyopathy in heterozygous plakoglobin-deficient mice. Circulation. 2006;114(17): 1799806. doi: 10.1161/CIRCULATIONAHA. 106. 624502

10. Saberniak J, Hasselberg NE, Borgquist R, Platonov PG, Sarvari SI, Smith HJ, et al. Vigorous physical activity impairs myocardial function in patients with arrhythmogenic right ventricular cardiomyopathy and in mutation positive family members. Eur J Heart Fail. 2014;16(12):1337-44. doi: 10.1002/ejhf.181

11. Möhlenkamp S, Lehmann N, Breuckmann F, Bröcker-Preuss M, Nassenstein K, Halle M, et al. Running: the risk of coronary events: prevalence and prognostic relevance of coronary atherosclerosis in marathon runners. Eur Heart J. 2008; 29(15): 1903-10. doi: 10.1093/eurheartj/ehn163

12. Benito B, Gay-Jordi G, Serrano-Mollar A, Guasch E, Shi Y, Tardif JC, Brugada J, et.al. Cardiac arrhythmogenic remodeling in a rat model of long-term intensive exercise training. Circulation 2011;123(1):13-22. doi: 10.1161/CIRCULATIONAHA. 110.938282

13. Guasch E, Benito B, Qi X, Cifelli C, Naud P, Shi Y, et al. Atrial fibrillation promotion by endurance exercise: demonstration and mechanistic exploration in an animal model. J Am Coll Cardiol. 2013; 62(1):68-77. doi: 10.1016/j.jacc.2013.01.091

14. Calvo N, Ramos P, Montserrat S, Guasch E, Coll-Vinent B, Domenech M, et al. Emerging risk factors and the dose-response relationship between physical activity and lone atrial fibrillation: a prospective case-control study. Europace 2016;18(1): 57-63. doi: 10.1093/europace/euv216

15. Armstrong MEG, Green J, Reeves GK, Beral V, Cairns BJ; Million Women Study Collaborators. Frequent physical activity may not reduce vascular disease risk as much as moderate activity: large prospective study of women in the United Kingdom. Circulation. 2015;131(8):721-9. doi: 10.1161/ CIRCULATIONAHA.114.010296

16. Priori SG, Aliot E, Blomstrom-Lundqvist C, Bossaert L, Breithardt G, Brugada P, et al. Task Force on Sudden Cardiac Death of the European Society of Cardiology. Eur Heart J. 2001;22(16): 1374-450. doi: 10.1053/euhj.2001.2824

17. Corrado D, Basso C, Rizzoli G, Schiavon M, Thiene G. Does sports activity hance the risk of sudden death in adolescents and young adults? J Am Coll Cardiol. 2003;42(11):1959-63. doi: 10.1016/j.jacc. 2003.03.002

18. Corrado D, Basso C, Schiavon M, Thiene G. Screening for hypertrophic cardiomyopathy in young athletes. N Eng J Med. 1998; 339(6):364-9. doi: 10.1056/NEJM199808063390602

19. Maron BJ, Gohman TE, Aeppli D. Prevalence of sudden cardiac death during competitive sports acti- vities in Minnesota high school athletes. J Am Coll Cardiol. 1998; 32(7):1881-4. doi: 10.1016/S07351097(98)00491-4

20. Maron BJ, Doerer JJ, Haas TS, Tierney DM, Mueller FO. Sudden deaths in young competitive athletes: analysis of 1866 deaths in the United States, 1980-2006. Circulation 2009;119(8):1085-92. doi: 10.1161/CIRCULATIONAHA.108.804617

21. Instituto Nacional de Estadística. El Uruguay en cifras 2011 [Internet]. Montevideo: INE; 2011 [consulta 21 Ago 2018]. Disponible en: www.ine.gub.uy/documents/10181/39317/Uruguay+en+cifras +2011.pdf

22. Maron BJ, Shirani J, Poliac LC, Mathenge R, Roberts WC, Mueller FO. Sudden death in young competitive athletes: clinical, demographic, and pathological profiles. JAMA 1996;276 (3): 199-204. doi: 10.1001/jama.1996.03540030033028

23. Maron BJ. Sudden death in young athletes. N Engl J Med. 2003; 349(11):1064-75. doi: 10.1056/ NEJMra 022783

24. Maron BJ, Carney KP, Lever HM, Lewis JF, Barac I, Casey SA, et al. Relationship of race to sudden cardiac death in competitive athletes with hypertrophic cardiomyopathy. J Am Coll Cardiol. 2003;41(6):974-80. doi: 10.1016/S0735-1097(02)02976-5

25. Suárez-Mier MP, Aguilera B. Causas de muerte súbita asociada al deporte en España. Rev Esp Cardiol [Internet]. 2002 Abr [consulta 28 May 2020]; 55(4): [aprox.9p.]. Disponible en: https://www.revesp-cardiol.org/en-linkresolver-causas-muerte-subita-asociada-al-13029695

26. Boraita A. Muerte súbita y deporte. ¿Hay alguna manera de prevenirla en los deportistas? Rev Esp Cardiol [Internet]. 2002 Abr [consulta 28 May 2020];55(4): [aprox. 3p]. Disponible en: https://www. revespcardiol.org/es-muerte-subita-deporte-hay-alguna-articulo-13029693

27. Aengevaeren VL, Hopman MTE, Thompson PD, Bakker EA, George KP, Thijssen DHJ, et al. Exercise-induced cardiac troponin I increase and incident mortality and cardiovascular events. Circulation 2019;140(10):804-14. doi: 10.1161/CIRCULATION AHA.119. 041627

28. Shave R, Baggish A, George K, Wood M, Scharhag J, Whyte G, et al. Exercise-induced cardiac troponin elevation: evidence, mechanisms, and implications. J Am Coll Cardiol. 2010;56 (3): 169-76. doi: 10.1016/j.jacc.2010.03.037

29. Furlanello F, Serdoz LV, Cappato R, De Ambroggi L. Illicit drugs and cardiac arrhythmias in athletes. Eur J Cardiovasc Prev Rehabil. 2007; 14(4):487-94. doi: 10.1097/HJR.0b013e3280ecfe3e

30. Desfibriladores externos automáticos Ley 18.360 (Set 26, 2008). Disponible en: https://www.laboratorioroig.com.uy/sites/default/files/pdf/18360.pdf 
31. Decreto 330/009 Reglamentación sobre adquisición y uso de desfibriladores externos automáticos (DEA) [Internet]. Montevideo: MSP; 2009 [consulta 23 Jul 2018]. Disponible en: http://www.impo.com.uy/bases/decretos/330-2009

32. Maron BJ, Thompson PD, Puffer JC, McGrew CA, Strong WB, Douglas PS, et al. Cardiovascular preparticipation screening of competitive athletes. A statement for health professionals from the Sudden Death Committee (clinical cardiology) and Congenital Cardiac Defects Committee (cardiovascular disease in the young), American Heart Association. Circulation 1996;94(4):850-6. doi: 10.1161/ 01.CIR.94.4.850

33. Maron BJ, Thompson PD, Puffer JC, McGrew CA, Strong WB, Douglas PS, et.al. Cardiovascular preparticipation screening of competitive athletes: addendum: an addendum to a statement for health professionals from the Sudden Death Committee (Council on Clinical Cardiology) and the Congenital Cardiac Defects Committee (Council on Cardiovascular Disease in the Young), American Heart Association. Circulation 1998;97(22):2294. doi 10.1161/01.CIR.97.22.2294

34. Maron BJ, Shirani J, Poliac LC, Mathenge R, Boberts WC, Mueller FO. Sudden death in young competitive athletes. Clinical, demographics, and pathological profiles. JAMA 1996;276(3):199-204. doi: 10.1001/jama.1996.03540030033028

35. Corrado D, Pelliccia A, Bjørnstad HH, Vanhees L, Biffi A, Borjesson M, et al. Cardiovascular pre-participation screening of young competitive athletes for prevention of sudden death: proposal for a common European protocol. Consensus Statement of the Study Group of Sport Cardiology of the Working Group of Cardiac Rehabilitation and Exercise Physiology and the Working Group of Myocardial and Pericardial Diseases of the European Society of Cardiology. Eur Heart J. 2005;26(5):516-24. doi 10.1093/eurheartj/ehi108
36. Corrado D, Basso C, Pavei A, Michieli P, Schiavon $\mathbf{M}$, Thiene G. Trends in sudden cardiovascular death in young competitive athletes after implementation of a preparticipation screening program. JAMA. 2006; 296 (13):1593-601. doi: 10.1001/ jama.296.1 3.1593

37. Maron BJ, Thompson PD, Ackerman MJ, Balady G, Berger S, Cohen D, et al. Recommendations and considerations related to preparticipation screening for cardiovascular abnormalities in competitive athletes: 2007 update: a scientific statement from the American Heart Association Council on $\mathrm{Nu}-$ trition, Physical Activity, and Metabolism: endorsed by the American College of Cardiology Foundation. Circulation 2007;115(12):1643-55. doi: 10.1161/ CIRCULATIONAHA.107.181423

38. Mont L, Pelliccia A, Sharma S, Biffi A, Borjesson M, Terradellas JB, et al. Pre-participation cardiovascular evaluation for athletic participants to prevent sudden death: Position paper from the EHRA and the EACPR, branches of the ESC. Endorsed by APHRS, HRS, and SOLAECE. Europace 2017;19(1):139-63. doi: 10.1093/europace/euw243

39. Harmon KG, Zigman M, Drezner JA. The effectiveness of screening history, physical exam, and ECG to detect potentially lethal cardiac disorders in athletes: a systematic review/meta-analysis. J Electrocardiol. 2015;48(3):329-38. doi: 10.1016/j.jelectrocard. 2015.02 .001

40. Corrado D, Pelliccia A, Heidbuchel H, Sharma S, Link M, Basso C, et al. Recommendations for interpretation of 12-lead electrocardiogram in the athlete. Eur Heart J. 2010;31(2):243-59. doi: 10.1093/ eurheartj/ehp473

41. Drezner JA, Ackerman MJ, Anderson J, Ashley E, Asplund CA, Baggish AL, et al. Electrocardiographic interpretation in athletes: the 'Seattle criteria'. Br J Sports Med. 2013;47(3):122-4. doi: 10.1136/ bjsports-2012-092067 